\title{
Delayed gadolinium-enhanced MRI of meniscus (dGEMRIM) and cartilage (dGEMRIC) in healthy knees and in knees with different stages of meniscus pathology
}

\author{
Ulf Sigurdsson ${ }^{1 *}$, Gunilla Müller ${ }^{2}$, Carl Siversson ${ }^{3}$, Eveliina Lammentausta ${ }^{4}$, Jonas Svensson ${ }^{5}$, \\ Carl-Johan Tiderius $^{6}$ and Leif E. Dahlberg ${ }^{6}$
}

\begin{abstract}
Background: Lesions in the meniscus are risk factors for developing knee osteoarthritis $(O A)$, not least because of the role of the meniscus in the pathological progression of OA. Delayed gadolinium enhanced MRI of cartilage (dGEMRIC) has extensively been used to identify pre-radiographic cartilage changes in OA. In contrast, its counterpart with regard to examination of the meniscus, gadolinium enhanced MRI of meniscus (dGEMRIM), has been less utilized. In this study we use 3D dGEMRIM in patients with meniscus lesions and compare them with previous results of healthy individuals.
\end{abstract}

Methods: Eighteen subjects with MRI-verified posteromedial meniscus lesions and 12 healthy subjects with non-injured and non-symptomatic knee joints, together 30 volunteers, were examined using 3D Look-Locker sequence after intravenous injection of Gd-DTPA ${ }^{2-}(0.2 \mathrm{mmol} / \mathrm{kg}$ body weight). Relaxation time (T1) was measured in the posterior meniscus and femoral cartilage before and 60,90,120 and 180 min after injection. Relaxation rate (R1 =1/T1) and change in relaxation rate $(\Delta R 1)$ were calculated. For statistical analyses, Student's $t$-test and Analysis of Variance (ANOVA) were used.

Results: The pre-contrast diagnostic MRI identified two sub-cohorts in the 18 patients with regard to meniscus injury: 1) 11 subjects with MRI verified pathological intrameniscal changes (grade 2 ) in the posteromedial meniscus only and no obvious cartilage changes. The lateral meniscus showed no pathology. 2) 7 subjects with MRI verified pathological rupture (grade 3) of the posteromedial meniscus and pathological changes in the lateral meniscus and/or medial and lateral joint cartilage.

Comparisons of pathological and healthy posteromedial meniscus revealed opposite patterns in both $T 1_{\mathrm{Gd}}$ and $\Delta \mathrm{R} 1$ values between pathological meniscus grade 2 and grade 3 . The concentration of the contrast agent was lower than in healthy meniscus in grade 2 lesions $(p=0.046)$ but tended to increase in grade 3 lesions $(p=0.110)$. Maximum concentration of contrast agent was reached after $180 \mathrm{~min}$ in both cartilage and menisci (except for grade 3 menisci where the maximum concentration was reached after $90 \mathrm{~min}$ ).

(Continued on next page)

\footnotetext{
* Correspondence: ulf.sigurdsson@skane.se

${ }^{1}$ Department of Orthopaedics, Lund University, Skåne University Hospital,

SE-205 02 Malmö, Sweden

Full list of author information is available at the end of the article
} 
(Continued from previous page)

Conclusion: dGEMRIM and dGEMRIC may be feasible to combine in vivo, preferably with one examination before and one $2 \mathrm{~h}$ after contrast injection. Possible different dGEMRIM patterns at different stages of meniscus lesions must be taken into account when evaluating meniscus pathology.

Keywords: dGEMRIC, dGEMRIM, Glycosaminoglycans, Meniscus, Cartilage

\section{Background}

Osteoarthritis (OA) affects approximately $10 \%$ of the elderly and is a leading cause of disability [1]. OA is suggested to be a whole-joint disease involving cartilage, ligaments, periarticular muscles, subchondral bone and menisci [2]. The meniscus has a critical protective role for knee joint integrity by absorbing impact and distributing load $[3,4]$. Overall, it is estimated that the knee meniscus carries 45 to $75 \%$ of the total joint load [5]. Loss of meniscal function has been strongly associated with development and progression of radiographic OA $[6,7]$. However, OA-related changes observed by radiography are late events in the degenerative process $[8,9]$. In $\mathrm{OA}$, the cartilage matrix undergoes pathological molecular cleavage with loss of type II collagen and glycosaminoglycans (GAG) $[1,10]$. To increase our understanding of pathogenic mechanisms in the early stage of the disease, anterior cruciate ligament and meniscus injured patients, with increased risk for OA, may serve as models $[6,11]$. Delayed gadolinium enhanced magnetic resonance imaging of cartilage (dGEMRIC) is an imaging technique that can identify pre-radiographic degenerative changes in articular cartilage, and potentially also in the meniscus $[12,13]$. When this technique is applied to the latter tissue, it is designated gadolinium enhanced MRI of meniscus (dGEMRIM). In dGEMRIC, the fixed charged density of GAG is studied in vivo through quantitative measurement of the longitudinal relaxation time (T1) of the articular cartilage in the presence of the negatively charged contrast agent Gd-DTPA ${ }^{2-}$ (gadolinium diethylene triaminepentaacetic acid). Recently, we have shown in healthy subjects that the contrast medium distributes also into the meniscus after intravenous injection (dGEMRIM) [13]. The purpose of our study is to evaluate dGEMRIM as a diagnostic tool in menisci with MRI verified pathological changes. The literature has indicated conflicting results concerning the GAG content in pathological meniscus [14-18]. Since these results may differ depending on the grade of pathological changes we have two separate main hypotheses. Firstly, we assume that meniscus with pathological grade 2 changes will display values suggesting an increased content of GAG compared to healthy meniscus and secondly, we assume that meniscus with pathological grade 3 changes will display values suggesting a decreased content of GAG compared to healthy meniscus. The specific aims of the study were first to investigate whether there is a measurable uptake of contrast agent in pathological menisci and when the maximum level of contrast agent was obtained, as measured in minutes after contrast injection. Second, we investigate whether there is a difference between the uptake of contrast agent in the posteromedial pathological meniscus and the posteromedial healthy meniscus in a previously examined knee without symptoms.

\section{Methods \\ Subjects}

In our study, we analyzed contrast enhanced MRI images of one knee in each of 30 volunteers. 12 of them were included in a prior study of contrast-enhanced MRI of healthy articular cartilage and meniscus of the knee [13]. Inclusion criteria for these 12 subjects (5 males, age 23-28 years, mean 25 years) were: 1 ) no history of injury or pain of the knee; 2) no abnormality at physical examination of the knee; 3) no pathologic changes at diagnostic MRI. 18 subjects with knee pain (experienced for a period of more than two months) were recruited by reviewing medical records. The knee pain was either due to spontaneous appearance or to a minor pivoting trauma and they had an MRI-verified posteromedial meniscus injury.

Exclusion criteria for all 30 subjects were: 1) contraindications for MRI (i.e. metal prosthesis, claustrophobia); 2) history of previous reactions to contrast agent and 3) medical record of kidney pathology.

\section{Contrast enhanced MRI}

The contrast enhanced MRI examination was performed within 4-6 months from the first diagnostic MRI. Prior to the intravenous injection of Gd-DTPA ${ }^{2-}$ (Magnevist $^{\circ}$, Bayer Schering Pharma Ag, Berlin, Germany), a diagnostic and quantitative T1 measurement examination was performed in the 18 subjects with injured meniscus. Meniscus changes were graded according to the classification of Lotysch [19]. Grade 1 changes represent one or several punctate signal intensities at one slice $(3 \mathrm{~mm}$ between the slices), grade 2 changes represent a linear (i.e. observed in several slices) intrameniscal signal intensity without extension to the articular surface and grade 3 changes represent a signal intensity extended to at least one articular surface.

Double dose of Gd-DTPA ${ }^{2-}(0.2 \mathrm{mmol} / \mathrm{kg}$ body weight $)$ was given in an antecubital vein. The time-point zero was 
set at the end of the drug injection. After injection, the subjects walked for ten minutes to optimize the distribution of Gd-DTPA ${ }^{2-}$ into the meniscus and cartilage. Subsequent examinations were performed at four time points (60, 90, 120 and $180 \mathrm{~min}$ ) after the injection. Data was collected using a 1.5 T MRI scanner (Siemens Sonata) with a dedicated knee coil. A three dimensional (3D) LookLocker sequence (field of view (FOV) $160 \times 160$, Matrix $256 \times 256$, 30 slices, slice thickness: $3 \mathrm{~mm}$, repetition time (TR) $2500 \mathrm{~ms}$, Flip Angle $6^{\circ}, 10$ inversion times (TIs) was used to acquire all 3D T1 maps. T1was calculated using the Pre-calculated Flip Angle Correction method and the associated flip angle slab profile was acquired from previous phantom measurements [20]. All data were evaluated using software programmed in MATLAB (The MathWorks Inc., Natick, MA, USA).

For the contrast-enhanced analysis, a radiologist used the pre-contrast MRI examination to define the location of the pathological meniscus changes. From that location, two sagittal slices, one in the lateral and one in the medial compartment, were selected from the 3D volume to enable analysis of the damaged posterior horn of the medial meniscus and the corresponding posterior part of the lateral meniscus. The same slices were used to examine the femoral articular cartilage in order to evaluate the optimal time point for combined analysis of cartilage and meniscus. Four ROIs were drawn in each knee to cover the posterior portion of the medial and lateral meniscus as well as the overlying femoral articular cartilage (Fig. 1) [21]. To standardize the procedure, all ROIs were drawn by a single investigator (US).

An average medial and lateral meniscus and corresponding cartilage pre- and post-contrast $\left(\mathrm{T} 1_{\mathrm{Pre}}, \mathrm{T} 1_{\mathrm{Gd}}\right)$ value was calculated for each of the four ROIs. The $\Delta \mathrm{R} 1$ value, which reflects the concentration of Gd-DTPA ${ }^{2-}$ in the tissue, was calculated using the formula $\Delta \mathrm{R} 1=1 / \mathrm{T} 1_{\mathrm{Gd}}-1 / \mathrm{T} 1_{\text {Pre }}$.

For statistical evaluation Student's $t$-test (when comparing cohorts at a specific time point) and analysis of variance (ANOVA) (when comparing cohorts over time) were used. The results are presented in the figures as mean values and with $95 \%$ confidence interval (95\% CI). A $p$-value of $<0.05$ was considered statistically significant.

All the subjects volunteered to participate in the study. All subjects were orally and by letter informed and a written consent was obtained. The study was approved by the ethics review board in Lund, Sweden.

\section{Results}

\section{Characterization of subjects}

The pre-contrast diagnostic MRI identified two subcohorts within the 18 patients with meniscus injury. 11 subjects (7 males, age 31-52 years, mean 40 years) had MRI verified pathological intrameniscal changes (grade 2) in the posteromedial meniscus only and no obvious

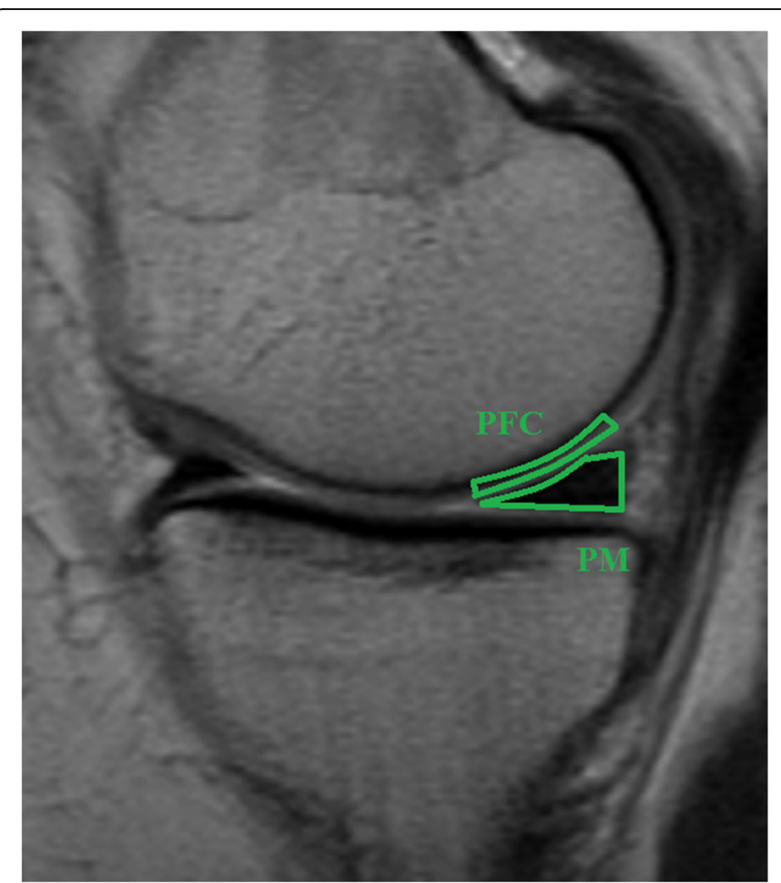

Fig. 1 Sagittal view of a knee joint. The average T1 value was calculated in the following regions of interest: PFC (Posterior Femoral Cartilage) and PM (Posterior Meniscus)

cartilage changes. The lateral meniscus showed no pathology. In the other 7 subjects (5 males, age 36-63 years, mean 54 years) the MRI examination showed a pathological rupture (grade 3 ) of the posteromedial meniscus and concomitant pathological changes in the lateral meniscus and/ or medial and lateral joint cartilage.

\section{$\mathrm{T} 1_{\text {Pres }} \mathrm{T}_{\mathrm{Gd}}$, and $\Delta \mathrm{R} 1$ values of healthy and pathological posteromedial meniscus}

Subjects with grade 2 lesions showed no difference in $\mathrm{T} 1_{\text {Pre }}$ values compared to subjects with healthy meniscus $(p=0.189, t$-test $)$. However, subjects with grade 3 lesions had longer $\mathrm{T} 1_{\text {Pre }}$ values than subjects with healthy meniscus $(p<0.001, t$-test) (Fig. $2 \mathrm{a}$ and Table 1$)$. The contrast medium diffused gradually into the menisci, with a maximum level observed after $180 \mathrm{~min}$. The maximum contrast level for grade 3 menisci was $90 \mathrm{~min}$. Subjects with grade 2 lesions had longer $\mathrm{T} 1_{\mathrm{Gd}}$ values than subjects with healthy meniscus ( $p=0.027$, ANOVA) (Fig. 2a and Table 1). There was no difference in $\mathrm{T} 1_{\mathrm{Gd}}$ in the posteromedial meniscus between subjects with meniscus lesions grade 3 and subjects with healthy meniscus ( $p=$ 0.315, ANOVA) (Fig. 2a and Table 1).

The concentration of the contrast medium, reflected by $\Delta \mathrm{R} 1$, was lower in meniscus with grade 2 lesion than in healthy meniscus ( $p=0.046$, ANOVA). Meniscus with grade 3 lesions tend to have higher concentration 
compared to meniscus in subjects with healthy knee joints ( $p=0.110$, ANOVA) (Fig. $2 \mathrm{~b}$ and Table 1 ).

\section{Temporal and spatial contrast distribution patterns of} $\mathrm{T} 1_{\text {Pre, }} \mathrm{T} 1_{\mathrm{Gd}}$, and $\Delta \mathrm{R} 1$ values in posterior articular cartilage The maximum level of contrast medium in the femoral cartilage was observed after 120-180 min (data not shown). Within knees with a medial meniscus lesion grade 2, mean $\mathrm{T} 1_{\mathrm{Pre}}, \mathrm{T} 1_{\mathrm{Gd}}$, and $\Delta \mathrm{R} 1$ did not differ in medial and lateral femoral cartilage. Further, no difference in $\mathrm{T} 1_{\mathrm{Pre}}, \mathrm{T} 1_{\mathrm{Gd}}$, and $\Delta \mathrm{R} 1$ values was found in lateral femoral cartilage in subjects with a medial meniscus

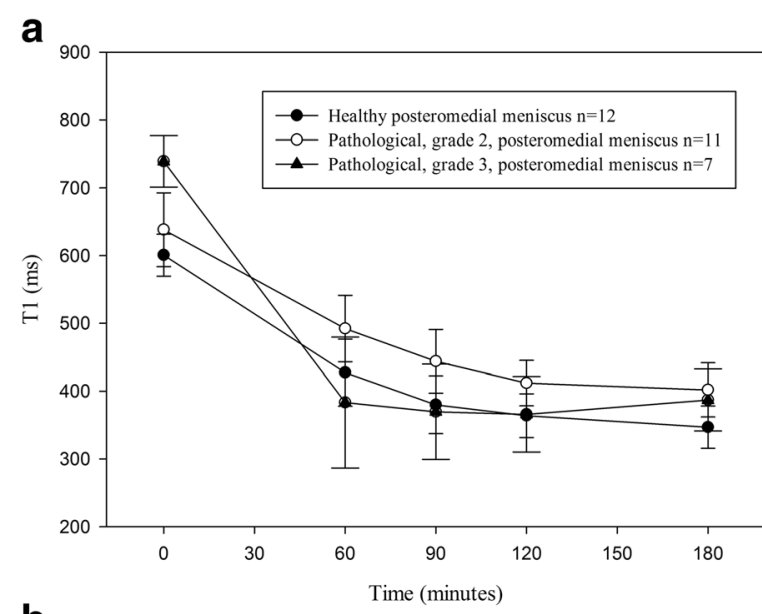

b

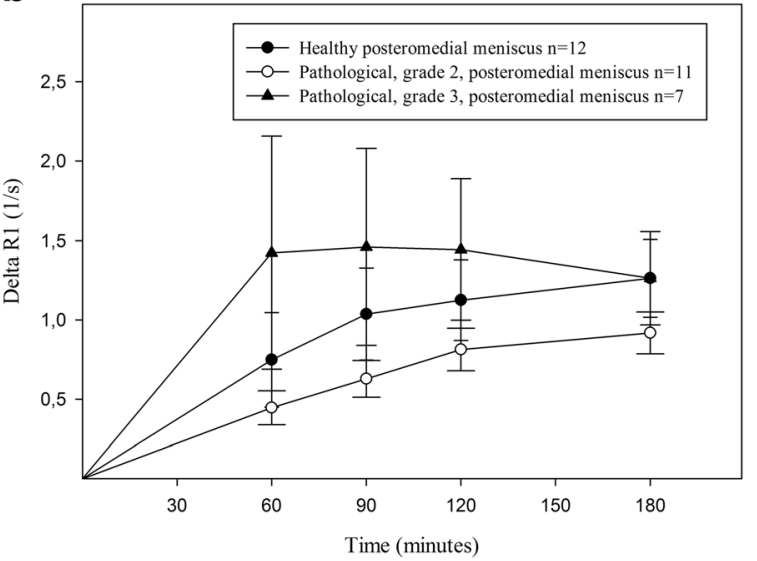

Fig. 2 a $T 1_{\text {pre }}$ and $T 1_{G d}$ values after intravenous injection of GdDTPA $^{2-}(0.2 \mathrm{mmol} / \mathrm{kg}$ body weight), in posteromedial healthy $(n=12$; closed circles), pathological grade 2 ( $n=11$; open circles) and pathological grade 3 ( $n=7$; closed triangles) menisci. $T 1_{\mathrm{Gd}}$ was longer in the pathological grade 2 menisci ( $p=0.027$, ANOVA) and showed no difference in the pathological grade 3 menisci compared to the healthy menisci. b $\Delta R 1$ values in posteromedial healthy $(n=12$; closed circles), pathological grade 2 ( $n=11$; open circles) and pathological grade 3 ( $n=7$; closed triangles) menisci, after an intravenous injection of Gd-DTPA ${ }^{2-}(0.2 \mathrm{mmol} / \mathrm{kg}$ body weight). The values were lower in the pathological grade 2 menisci $(p=0.046$, ANOVA) and tend to show higher values in the pathological grade 3 menisci ( $p=0.110$, ANOVA) compared to the healthy menisci injury grade 2 and subjects with healthy knee joints (data not shown). In the medial femoral cartilage, $\mathrm{T} 1_{\mathrm{Gd}}$, but not $\mathrm{T} 1_{\text {Pre }}$ or $\Delta \mathrm{R} 1$, was longer in subjects with meniscus injury grade 2 compared to subjects with healthy knee joints $(p=0.027$, ANOVA, $p=0.159, t$-test and $p=$ 0.280 , ANOVA). The compartments with grade 3 lesions had cartilage changes that were severe enough to compromise the $\mathrm{T} 1_{\mathrm{Gd}}$ calculations. These results were therefore not evaluated.

\section{Discussion}

In our study we show that dGEMRIM can differentiate between pathological (grade 2) and healthy meniscal tissue. The decreased distribution of Gd-DPTA ${ }^{2-}$ in the pathological menisci grade 2 suggests more negative charge and an increased GAG concentration in this meniscus pathology. These results are consistent with previous meniscus studies that showed increased GAG concentration by histology and biochemical analysis [14, 15]. In addition, it has also been reported that proteoglycan content $(\mu \mathrm{g} / \mathrm{mg}$ dry weight) in menisci with meniscal tears increase in relation to the severity of the meniscal degeneration [16]. Interestingly, the proteoglycan content in the meniscus does not seem to increase in rheumatoid arthritis [22].

A previous dGEMRIM study of 17 subjects with knee symptoms and MRI verified pathology in cartilage and menisci, showed a trend towards decreased $\mathrm{T} 1_{\mathrm{Gd} 90}$ values (i.e. values obtained $90 \mathrm{~min}$ after injection of contrast agent) in pathologically changed meniscus (posteromedial grade $2 ; n=5$ ) compared to healthy meniscus within the same knee joint [17]. All patients in that study were diagnosed with OA (Kellgren \& Lawrence grade 1 or 2) and the results are in agreement with the results for the meniscus tissue with pathological changes grade 3 in our study. $\Delta R 1$ values, not presented in the study by van Tiel et al., may have enabled a more thorough comparison with our study. It can be speculated that increased GAG synthesis, reflected by longer $\mathrm{T} 1_{\mathrm{Gd}}$ and decreased $\Delta \mathrm{R} 1$ values for pathological meniscus grade 2 in knee joints without cartilage damages in our study, is an early event in meniscus pathology. In contrast, in later stages of degenerative disease as presented in our study by pathological meniscus grade 3 and in the study by van Tiel et al. (knee joints with meniscus changes grade 2 and diagnosed OA, Kellgren \& Lawrence grade 1 or 2), a more extensive damage to the knee joint results in a facilitated diffusion of contrast medium into the meniscus with corresponding shorter $\mathrm{T} 1_{\mathrm{Gd}}$ and increased $\Delta \mathrm{R} 1$.

In our study the maximum level of contrast medium in healthy femoral cartilage occurred approximately $120 \mathrm{~min}$ after injection. This is consistent with earlier results [23].

Comparing the femoral cartilage between healthy knee joints and knee joints with pathological grade 2 changes 
Table $1 \mathrm{~T} 1_{\text {Pre, }} \mathrm{T} 1_{\mathrm{Gd}}$ and $\Delta \mathrm{R} 1$ values in healthy $(n=12)$, pathological grade $2(n=11)$ and pathological grade $3(n=7)$ posteromedial menisci

\begin{tabular}{|c|c|c|c|c|c|c|c|c|c|c|c|c|c|c|c|}
\hline \multicolumn{16}{|c|}{ T1Pre,T1Gd and $\Delta$ R1 values in healthy and pathological- grade 2 and 3- posteromedial meniscus } \\
\hline \multirow[t]{2}{*}{ Time (min) } & \multicolumn{3}{|c|}{ Precontrast (Mean(Cl) T1Pre (ms)) } & \multicolumn{3}{|l|}{60} & \multicolumn{3}{|l|}{90} & \multicolumn{3}{|l|}{120} & \multicolumn{3}{|l|}{180} \\
\hline & Healthy & Grade 2 & Grade 3 & Healthy & Grade 2 & Grade 3 & Healthy & Grade 2 & Grade 3 & Healthy & Grade 2 & Grade 3 & Healthy & Grade 2 & Grade 3 \\
\hline Mean(Cl) T1Gd (ms) & $601 \pm 31$ & $638 \pm 54$ & $739 \pm 38$ & $427 \pm 49$ & $492 \pm 49$ & $383 \pm 96$ & $379 \pm 43$ & $444 \pm 46$ & $370 \pm 70$ & $364 \pm 32$ & $412 \pm 34$ & $366 \pm 55$ & $347 \pm 31$ & $402 \pm 40$ & $387 \pm 45$ \\
\hline$p$ values & & 0,189 & $<0,001$ & & 0,052 & 0,307 & & 0,036 & 0,761 & & 0,032 & 0,936 & & 0,024 & 0,106 \\
\hline$\%$ of difference & & 6 & 23 & & 15 & 11 & & 17 & $<1$ & & 13 & $<1$ & & 16 & 12 \\
\hline $\operatorname{Mean}(\mathrm{Cl}) \Delta \mathrm{R} 1$ (1/s) & & & & $0,75 \pm 0,30$ & $0,47 \pm 0,17$ & $1,42 \pm 0,73$ & $1,04 \pm 0,29$ & $0,70 \pm 0,18$ & $1,46 \pm 0,62$ & $1,13 \pm 0,25$ & $0,86 \pm 0,20$ & $1,44 \pm 0,44$ & $1,26 \pm 0,25$ & $0,93 \pm 0,22$ & $1,26 \pm 0,30$ \\
\hline$p$ values & & & & & 0,099 & 0,031 & & 0,044 & 0,120 & & 0,089 & 0,138 & & 0,041 & 0,997 \\
\hline$\%$ of difference & & & & & 60 & 89 & & 49 & 37 & & 31 & 27 & & 35 & $<1$ \\
\hline
\end{tabular}


in the posteromedial meniscus, we found no differences with regard to the $\mathrm{T} 1_{\mathrm{Pre}}, \mathrm{T} 1_{\mathrm{Gd}}$ and $\Delta \mathrm{R} 1$ values on the lateral side. On the medial side, there was a difference in $\mathrm{T} 1_{\mathrm{Gd}}$ but not in the $\mathrm{T} 1_{\text {Pre }}$ or $\Delta \mathrm{R} 1$ values. These findings strengthen the results from the diagnostic MRI, suggesting that the posteromedial meniscus is the only tissue in the examined knee joint with pathological meniscus changes grade 2 that has pathological changes.

It is important to acknowledge that other factors than GAG content may contribute to the distribution of contrast agent into a specific tissue. The fact that the wet weight GAG concentration in the meniscus is $0.3 \%$ as opposed to $2.0 \%$ in articular cartilage [24] and that collagen network differs between the two tissues [25, 26], suggests that GAG content is not the only factor that determines the contrast distribution into the meniscus. Previous studies $[18,27]$ addressed this issue in more detail using both an ionic (inversely related to the GAG content) and a non-ionic (no known interaction with GAG content) contrast agent when investigating meniscus and femoral cartilage in knee joints with OA. Data from these studies demonstrated similar differences in $\mathrm{T} 1_{\mathrm{Gd}}$ values in meniscus and cartilage when comparing subjects with $\mathrm{OA}$ and healthy volunteers, with both kinds of contrast agents, indicating that GAG is not the sole factor influencing the uptake of contrast agent. The authors of these studies speculated that the integrity of the collagen network may play a role, as well as increased diffusion due to degenerative changes of both the meniscus and cartilage.

Using dGEMRIC, Hawezi et al. [28] showed a depthwise variation in contrast distribution in femoral knee cartilage. The maximum concentration of the contrast agent was observed at different time points in different cartilage layers: at 120-180 $\mathrm{min}$ in the superficial layer and at $240 \mathrm{~min}$ or more in the deeper layers. Furthermore, the differences increased with cartilage thickness. Although the GAG content and the composition of different collagen types differ when comparing different parts of the meniscus [23-25], it is unlikely that there is a larger depthwise alteration of the concentration of the contrast agent in the meniscus since the contrast agent diffuses into the meniscus from two sides. These authors further discussed a wash out effect of contrast agent occurring after approximately $120 \mathrm{~min}$. It has previously been demonstrated that following an intravenous Gd-DTPA ${ }^{2-}$ injection, the gadolinium concentration in blood plasma will reach its maximum and then decrease within $120 \mathrm{~min}$ [29]. Assuming similar concentration decay in synovial fluid, the gadolinium concentration of articular cartilage will be higher than that in synovial fluid and a wash out of gadolinium from the cartilage will start approximately after $120 \mathrm{~min}$. The wash out effect needs to be considered in order to choose an optimal time point post contrast to investigate pathological menisci with dGEMRIM. From our study as well as previous studies, it can be concluded that $120 \mathrm{~min}$ post contrast seems optimal for the evaluation of both meniscus and cartilage tissue [13].

It may be argued that the exclusion of grade 1 meniscus lesions is a limitation of the present study. However, grade 1 changes are very difficult to depict when drawing ROIs on a specific slice. It is therefore a significant risk that the measurements would be calculated on the wrong slice and resulting in incorrect values.

The results of menisci with pathological changes grade 3 may be an issue for discussion. Firstly, these changes are often considered as an advanced stage of OA and an examination with contrast enhanced MRI would in clinical use be of less interest. Furthermore, meniscal tears might fill with contrast agent directly from joint cavity, causing erroneously $\mathrm{T} 1_{\mathrm{Gd}}$ relaxation times. Therefore, this must be taken in consideration when evaluating the obtained values using dGEMRIM to examine menisci with pathological grade 3 changes.

An issue using gadolinium as contrast agent is the possible occurrence of nephrogenic systemic fibrosis (NSF). This has mainly been observed in patients with severe chronic or acute renal failure following exposure to gadolinium-based contrast agents [30]. However, after the introduction of clinical guidelines restricting the use of gadolinium in these patients, reports from several academic medical centers have been published indicating that no new cases of NSF have been observed among this category of patients [31, 32].

\section{Conclusions}

dGEMRIM and dGEMRIC may be feasible to combine in vivo, preferably with one examination before and one $2 \mathrm{~h}$ after contrast injection. Possible different dGEMRIM patterns at different stages of meniscus lesions must be taken into account when evaluating meniscus pathology.

\begin{abstract}
Abbreviations
3D: Three-dimensional; $\triangle R$ 1: Difference in R1, reflecting the Gd-DTPA ${ }^{2-}$ concentration; ANOVA: Analysis of variance; $\mathrm{Cl}$ : Confidence interval; dGEMRIC: delayed gadolinium-enhanced magnetic resonance imaging of cartilage; dGEMRIM: delayed gadolinium-enhanced magnetic resonance imaging of meniscus; FOV: Field of view; Gd-DTPA ${ }^{2-}$ : Gadolinium diethylene triaminepentaacetic acid; GAG: Glycosaminoglycan; MRI: Magnetic resonance imaging; NSF: Nephrogenic systemic fibrosis; OA: Osteoarthritis; R1: Relaxation rate, reflecting the Gd-DTPA ${ }^{2-}$ distribution; ROI: Region of interest; $\mathrm{T} 1$ : Longitudinal relaxation time; $\mathrm{T} 1_{\text {pre: }} \mathrm{T} 1$ relaxation time without Gd-DTPA ${ }^{2-}$ (pre-contrast); $T 1_{\mathrm{Gd}}: \mathrm{T1}$ relaxation time after saturation with GdDTPA $^{2-}$ (post-contrast); TI: Inversion time; TR: Repetition time
\end{abstract}

\section{Acknowledgements}

We would like to thank Jeanette Nilsson for help with administrative work (contacting and mailing the subjects) and Jan-Åke Nilsson for statistical advice (both employees are at Lund University, Sweden).

Funding

This study was supported by grants from the Swedish Research Council (VR), governmental funding of clinical research within the Swedish National Health Service (ALF), Skåne County Council's Research and Development 
Foundation (FoU), the Swedish National Centre for Reasearch in Sports (CIF), The Swedish Rheumatism Association (RF), Skåne University Hospital funds, King Gustaf V's 80-Year Fund, The Herman Järnhardt Foundation, The Alfred Österlund Foundation and Greta and Johan Kock's Foundation.

\section{Availability of data and materials}

The datasets analyzed during the current study are available from the corresponding author on reasonable request.

\section{Authors' contribution}

US wrote the manuscript. US, CJT and LED participated in the design of the study. GM identified and graded the meniscus lesions. CS and JS developed the pulse sequence used to acquire the 3D T1 maps and optimized the acquisition protocol. EL and CS developed the software to enable the analyses of the MRI images. US analyzed the MRI images and performed the statistical analysis. All the authors conceived the aspects of this study, and participated in its coordination. All authors read and approved the final manuscript.

\section{Competing interests}

Authors US, GM, EL, JS, CJT and LED declare that they have no competing interests. Author CS is an employee of Spectronic Medical AB (Helsingborg, Sweden).

\section{Consent for publication}

Not applicable.

\section{Ethics approval and consent to participate}

All the subjects volunteered to participate in the study. All subjects were orally and by letter informed and a written consent was obtained. The study was approved by the ethics review board in Lund, Sweden. The reference number for the ethical approval in this manuscript is: Dnr 446/2008.

\section{Author details}

'Department of Orthopaedics, Lund University, Skåne University Hospital, SE-205 02 Malmö, Sweden. ${ }^{2}$ Institute of Radiology und Scintigraphy, Kantonsspital Lucerne, Spitalstrasse, 6000 Lucerne, Switzerland. ${ }^{3}$ Medical Radiation Physics, Department of Translational Medicine, Lund University, Skåne University Hospital, SE-205 02 Malmö, Sweden. ${ }^{4}$ Department of Diagnostic Radiology, Oulu University Hospital, PO Box 50, Fl-90029 OYS Oulu, Finland. ${ }^{5}$ Medical Imaging and Physiology, Skåne University Hospital, SE-221 85 Lund, Sweden. ${ }^{6}$ Department of Orthopaedics, Clinical Sciences Lund, Lund University, Skåne University Hospital, SE-221 85 Lund, Sweden.

Received: 6 February 2016 Accepted: 8 September 2016

\section{Published online: 29 September 2016}

\section{References}

1. Busija L, Bridgett L, Williams SR, Osborne RH, Buchbinder R, March L, Fransen M. Osteoarthritis. Best Pract Res ClinRheumatol. 2010;24(6):757-68. Review.

2. Brandt KD, Radin EL, Dieppe PA, van de Putte L. Yet more evidence that osteoarthritis is not a cartilage disease. Ann Rheum Dis. 2006;65(10):1261-4.

3. Proctor CS, Schmidt MB, Whipple RR, Kelly MA, Mow VC. Material properties of the normal medial bovine meniscus. J Orthop Res. 1989;7(6):771-82.

4. Ghadially FN, Lalonde JM, Wedge JH. Ultrastructure of normal and torn menisci of the human knee joint. J Anat. 1983;136(Pt 4):773-91.

5. Shrive NG, O'Connor JJ, Goodfellow JW. Load-bearing in the knee joint. ClinOrthopRelat Res. 1978;131:279-87.

6. Neuman P, Englund M, Kostogiannis I, Fridén T, Roos H, Dahlberg LE. Prevalence of tibiofemoral osteoarthritis 15 years after nonoperative treatment of anterior cruciate ligament injury: a prospective cohort study. Am J Sports Med. 2008;36(9):1717-25.

7. Berthiaume MJ, Raynauld JP, Martel-Pelletier J, Labonté F, Beaudoin G, Bloch DA, Choquette D, Haraoui B, Altman RD, Hochberg M, Meyer JM, Cline GA, Pelletier JP. Meniscal tear and extrusion are strongly associated with progression of symptomatic knee osteoarthritis as assessed by quantitative magnetic resonance imaging. Ann Rheum Dis. 2005;64(4):556-63.

8. Boegård TRO, Petersson IF, Jonsson K. Joint-space width in the weightbearing radiogram of the tibiofemoral joint. Should the patient stand on one leg or two? ActaRadiol. 1998;39(1):32-5.

9. Davies AP, Calder DA, Marshall T, Glasgow MM. Plain radiography in the degenerate knee. A case for change. J Bone Joint Surg (Br). 1999;81(4):632-5.
10. Poole AR, Rizkalla G, lonescu M, Reiner A, Brooks E, Rorabeck C, Bourne R, Bogoch E. Osteoarthritis in the human knee: a dynamic process of cartilage matrix degradation, synthesis and reorganization. Agents Actions Suppl. 1993;39:3-13.

11. Pernin J, Verdonk P, Selmi TAS, Massin P, Neyret P. Long-term follow-up of 24.5 years after intra-articular anterior cruciate ligament reconstruction with lateral extra-articular augmentation. Am J Sports Med. 2010;38(6):1094-102.

12. Tiderius CJ, Jessel R, Kim YJ, Burstein D. Hip dGEMRIC in asymptomatic volunteers and patients with early osteoarthritis: the influence of timing after contrast injection. MagnResonMed. 2007;57:803-5.

13. Sigurdsson U, Siversson C, Lammentausta E, Svensson J, Tiderius CJ, Dahlberg LE. In vivo transport of Gd-DTPA2- into human meniscus and cartilage assessed with delayed gadolinium-enhanced MRI of cartilage (dGEMRIC). MusculoskeletDisord. 2014;15:226.

14. Sun Y, Mauerhan DR, Kneisl JS, James Norton H, Zinchenko N, Ingram J, Hanley Jr EN, Gruber HE. Histological examination of collagen and proteoglycan changes in osteoarthritic menisci. Open Rheumatol J. 2012;6:24-32.

15. Peters TJ, Smillie IS. Studies on the chemical composition of the menisci of the knee joint with special reference to the horizontal cleavage lesion. ClinOrthopRelat Res. 1972:86:245-52.

16. Herwig J, Egner E, Buddecke E. Chemical changes of human knee joint menisci in various stages of degeneration. Ann Rheum Dis. 1984;43(4):635-40.

17. van Tiel J, Kotek G, Reijman M, Bos PK, Bron EE, Klein S, Verhaar JA, Krestin GP, Weinans H, Oei EH. Delayed gadolinium-enhanced MRI of the meniscus (dGEMRIM) in patients with knee osteoarthritis: relation with meniscal degeneration on conventional MRI, reproducibility, and correlation with dGEMRIC. EurRadiol. 2014:24(9):2261-70.

18. Li W, Edelman RR, Prasad PV. Delayed contrast enhanced MRI of meniscus with ionic and non-ionic agents. J MagnReson Imaging. 2011;33(3):731-5.

19. Lotysch M, Mink J, Crues JV, Schwartz SA. Magnetic resonance imaging in the detection of meniscal injuries. MagnReson Imaging. 1986;4:94.

20. Siversson C, Tiderius CJ, Dahlberg L, Svensson J. Local Flip Angle Correction for Improved Volume T1-Qantification in Three-Dimensional dGEMRIC Using the Look-Locker Technique. J MagnReson Imaging. 2009;30:834-41.

21. Eckstein F, Ateshian G, Burgkart R, Burstein D, Cicuttini F, Dardzinski B, Gray M, Link TM, Majumdar S, Mosher T, Peterfy C, Totterman S, Waterton J, Winalski S, Felson D. Proposal for a nomenclature for magnetic resonance imaging based measures of articular cartilage in osteoarthritis. Osteoarthritis Cartilage. 2006;14(10):974-83.

22. Ghosh P, Ingman AM, Taylor TK. Variations in collagen, non-collagenous proteins, and hexosamine in menisci derived from osteoarthritic and rheumatoid arthritic knee joints. J Rheumatol. 1975;2(1):100-7.

23. Tiderius CJ, Olsson LE, de Verdier H, Leander P, Ekberg O, Dahlberg L. GdDTPA2)-enhanced MRI of femoral knee cartilage: a dose-response study in healthy volunteers. MagnReson Med. 2001;46(6):1067-71.

24. McNicol D, Roughley PJ. Extraction and characterization of proteoglycan from human meniscus. Biochem J. 1980;185(3):705-13.

25. Akeson WH, Amiel DA, Gershuni DH. Articular cartilage physiology and metabolism. In: Resnick D, editor. Diagnosis of bone and joint disorders. 3rd ed. Philadelphia: WB Saunders; 1995. p. 769-90.

26. McDermott ID, Sharifi F, Bull AM, Gupte CM, Thomas RW, Amis AA. An anatomical study of meniscal allograft sizing. Knee Surg Sports TraumatolArthrosc. 2004;12(2):130-5.

27. Li W, Scheidegger R, Wu Y, Edelman RR, Farley M, Krishnan N, Burstein D, Prasad PV. Delayed contrast-enhanced MRI of cartilage: comparison of nonionic and ionic contrast agents. MagnReson Med. 2010;64(5):1267-73.

28. Hawezi ZK, Lammentausta E, Svensson J, Dahlberg LE, Tiderius CJ. In vivo transport of Gd-DTPA2- in human knee cartilage assessed by depth-wise dGEMRIC analysis. J MagnReson Imaging. 2011;34(6):1352-8.

29. Weinmann HJ, Laniado M, Mützel W. Pharmacokinetics of GdDTPA dimeglumine after intravenous injection into healthy volunteers. PhysiolChem Phys Med NMR. 1984;16(2):167-72.

30. Grobner T. Gadolinium a specific trigger for the development of nephrogenic fibrosingdermopathy and nephrogenic systemic fibrosis? Nephrol Dial Transplant. 2006;21:1104-8.

31. Martin DR, Krishnamoorthy SK, Kalb B. Decreased incidence of NSF in patients on dialysis after changing gadolinium contrast-enhanced MRI protocols. J MagnReson Imaging. 2010;31:440-6.

32. Wang Y, Alkasab TK, Narin O. Incidence of nephrogenic systemic fibrosis after adoption of restrictive gadolinium-based contrast agent guidelines Radiology. 2011;260:105-11. 